\title{
The dysfunctional inner mirror: poor insight in obsessive- compulsive disorder, contributions to heterogeneity and outcome
}

\author{
Himani Kashyap, ${ }^{1,2 *}$ J. Keshav Kumar, ${ }^{1}$ Thennarasu Kandavel, ${ }^{3}$ and \\ Y. C. Janardhan Reddy ${ }^{2,4}$
}

\footnotetext{
${ }^{1}$ Neuropsychology Unit, Department of Clinical Psychology, National Institute of Mental Health and Neuro Sciences (NIMHANS), Bangalore, India

${ }^{2}$ Obsessive Compulsive Disorder Clinic, National Institute of Mental Health and Neuro Sciences (NIMHANS), Bangalore, India

${ }^{3}$ Department of Biostatistics, National Institute of Mental Health and Neuro Sciences (NIMHANS), Bangalore, India

${ }^{4}$ Department of Psychiatry, National Institute of Mental Health and Neuro Sciences (NIMHANS), Bangalore, India
}

Received 16 June 2015; Accepted 21 August 2015

Poor insight has long been considered a hallmark of psychosis, and has been the subject of much research in schizophrenia. Historically, preserved insight has been considered characteristic of obsessive-compulsive disorder (OCD). Insight in OCD refers to an awareness of the senselessness of obsessions, of the need for treatment, or more generally of the illness itself. Almost 2 decades ago, the Diagnostic and Statistical Manual of Mental Disorders, Fourth Edition (DSM-IV) field trial proposed the inclusion of a "poor insight" subtype "to alert clinicians to the range of insight that characterizes individuals with OCD ... [and] encourage researchers to explore the relationship among insight and other aspects of psychopathology and treatment." ${ }^{\text {T1 }}$ The DSM Fifth Edition (DSM-5) has now incorporated a range of qualifiers for insight, such as "good or fair insight," "poor insight," and "absent insight." The forthcoming International Classification of Diseases, 11th Revision (ICD-11) proposes to include similar qualifiers for insight. $^{2}$

It is now generally estimated that patients with poor insight form over one-quarter of the population of OCD. Poor insight has been associated with greater severity, earlier age at onset, longer duration of illness, and poorer treatment response, as well as obsessivecompulsive symptom dimensions such as hoarding and contamination, ${ }^{3-5}$ but there is only modest concordance among studies on specific associations. At the National Institute of Mental Health \& Neurosciences (NIMHANS)

* Address for correspondence: Himani Kashyap, Neuropsychology Department, The Melbourne Clinic, 130 Church Street, Richmond, VIC 3121, Australia.

(Email: dr.himanikashyap@gmail.com) in India, we have assessed insight in 148 individuals with DSM-IV OCD using the Brown Assessment of Beliefs Scale (BABS). Poorer insight correlated with greater severity of illness measured by the Yale-Brown Obsessive-Compulsive Scale (YBOCS), with contamination obsessions, washing compulsions, and comorbid social phobia predicting poorer insight, while mental rituals were associated with better insight. The relationship with contamination-washing symptoms is likely to be culture-specific, but has also been reported in a large multicenter study from Brazil. ${ }^{4}$ Contamination/washing symptoms are part of what have been described as "socioterritorial" or "security" concerns. In Indian culture, cleanliness, purity, and hygiene are almost synonymous, and are central tenets of Ayurveda-the traditional Indian system of medicine-along with being prerequisite to many religious practices. As a result, exaggerated concerns about cleanliness and an associated "Suchi bai" syndrome are considered culturally acceptable in certain parts of India. Hence it is likely that from the evolutionary, cultural, and subjective standpoints, contamination concerns are less likely to be considered irrational/excessive, even when present to a substantial degree, and are held with greater conviction and fixity.

It has been posited that poor insight may be merely an epiphenomenon of the severity of illness. However, across several studies, insight has consistently shown associations with specific clinical characteristics, even when severity is controlled for. The most puzzling aspect for clinicians over the years has been the strong pressure individuals feel to indulge in rituals despite the recognition that they are intrusive or time-consuming. Many individuals argue vehemently about the rationality of their symptoms, and also involve their families in their 
elaborate rituals. It is as if their "inner mirrors" are unable to process corrective feedback, unlike the mirror in the classic fairytale. Parallels to poor insight in psychosis and anosognosia (unawareness of deficits) following neurological lesions added to the mounting evidence for neurobiological factors underlying poor insight in OCD. A magnetic resonance imaging (MRI) investigation comparing individuals with good and poor insight in $\mathrm{OCD}^{6}$ demonstrated a greater frequency of abnormalities (83\% versus $21 \%$ ) in those with poor insight, with the basal ganglia and the parietal lobe being the most frequent sites of abnormalities. In our previously published study, ${ }^{7}$ we found that poor insight in OCD was linked to significantly poorer conflict resolution/response inhibition, verbal memory, and fluency. The only two other published neuropsychological studies have also shown worse performance on verbal memory, ${ }^{8,9}$ and on cognitive flexibility ${ }^{9}$ in poor insight compared to good insight OCD. Therefore, rather than simply being more severely ill, it is likely that some individuals tend to hold their obsessive beliefs with greater certainty even in the face of contradictory information due to specific cognitive deficits. Some possible reasons for this follow:

- Difficulties with conflict resolution/response inhibition may prevent them from adaptively resolving conflicts between the existing belief system and incoming corrective information.

- Memory problems may make it difficult to update their belief system with new information.

- Fluency problems impede their access to information that may exist in memory stores. ${ }^{7}$

These findings have treatment implications; beliefmodification-based cognitive behavioral therapy (CBT) may be more useful than just habituation-based CBT with poor insight OCD. It would also be interesting to examine if augmentation of selective serotonin reuptake inhibitors (SSRIs) with antipsychotics has any special role in treatment of poor insight OCD.

Poor insight is a newcomer in OCD phenomenology (relative to, for instance, poor insight in psychosis), and although it has been the focus of some attention as an important prognostic factor, there is still a long way to go in terms of a gold standard for its evaluation. Much of the discrepancy in research findings can be attributed to differences in the conceptualization and assessment of insight. The evaluation of insight has traditionally rested upon a one-dimensional, dichotomous categorization, usually whether or not the individual acknowledges that the concerns are exaggerated or unreasonable, and more detailed evaluations are often deemed unnecessary, particularly in busy clinical settings. However insight is rarely an all-or-none phenomenon, and may vary with time, as well as with different aspects of an obsessive belief; some beliefs may be held with more conviction than others. In this context, the use of an objective measure of insight that evaluates several dimensions/aspects of insight is important, both in routine clinical settings as well as research. For instance, studies using a single-item measure, such as the YBOCS Item 11 , classify between $9 \%$ and $16 \%$ of subjects as having poor insight, ${ }^{5,10}$ whereas those using the BABS or other measures of insight consistently identify a higher percentage, between $20 \%$ and $30 \%$ of subjects, as having poor insight. ${ }^{1,4}$ We have found in the course of our work that although individuals might acknowledge generally that they have OCD and require treatment, when they are questioned specifically about a core belief, they are more reluctant to consider alternate views. This has significant consequences on management plans and prognosis, especially since those with poor insight are less likely to themselves seek treatment or adhere to it, and are at risk of being misdiagnosed as having a psychotic disorder.

OCD has been going through a state of flux, as its place in the current nosological systems has been questioned, its interrelationships with other disorders such as impulse-control disorders and schizophrenia have been explored, and the inherent variability of the disorder with its many symptom dimensions have been more extensively investigated. It is likely that re-examination of traditional conceptions, along with further research on the neurobiology of insight, specific symptom dimensions, and relationships with anxiety and impulse-control disorders and schizophrenia, will contribute greatly to the current understanding of OCD.

\section{Disclosures}

The authors do not have anything to disclose.

\section{REFERENCES:}

1. Foa EB, Kozak MJ, Goodman WK, Hollander E, Jenike MA, Rasmussen SA. DSM IV field trial: obsessive-compulsive disorder. Am J Psychiatry. 1995; 152(1): 90-96.

2. Simpson HB, Reddy YC. Obsessive-compulsive disorder for ICD-11 proposed changes to the diagnostic gudelines and specifiers. Rev Bras Psiquiatr. 2014; 36(Suppl 1): 3-13.

3. Kishore VR, Samar R, Reddy YCJ, Chandrashekhar CR, Kandavel T. Clinical characteristics and treatment response in poor and good insight obsessive-compulsive disorder. Eur Psychiatry. 2004; 19(4): 202-208.

4. Jakubovski E, Pittenger C, Torres AR, et al. Dimensional correlates of poor insight in obsessive-compulsive disorder. Prog Neuropsychopharmacol Biol Psychiatry. 2011; 35(7): 1677-1681.

5. Cherian A, Narayanaswamy J, Srinivasaraju R, et al. Does insight have specific correlation with symptom dimensions in OCD? J Affect Disord. 2012; 138(3): 352-359. 
6. Aigner M, Zitterl W, Prayer D, et al. Magnetic resonance imaging in patients with obsessive-compulsive disorder with good versus poor insight. Psychiatry Res. 2005; 140(2): 173-179.

7. Kashyap H, Kumar JK, Kandavel T, Reddy YCJ. Neuropsychological correlates of insight in obessive compulsive disorder. Acta Psychiatr Scand. 2012; 126(2): 106-114.

8. Kitis A, Akdede BBK, Alptekin K, et al. Cognitive dysfunctions in patients with obsessive-compulsive disorder compared to the patients with schizophrenia: relation to overvalued ideas.
Prog Neuropsychopharmacol Biol Psychiatry. 2007; 31(1): 254-261.

9. Tumkaya S, Karadag F, Oguzhanoglu NK, et al. Schizophrenia with obsessive-compulsive disorder and obsessive-compulsive disorder with poor insight: a neuropsychological comparison. Psychiatry Res. 2009; 165(1-2): 38-46.

10. Catapano F, Sperandeo R, Perris F, Lanzro M, Maj M. Insight and resistance in patients with obsessive-compulsive disorder.

Psychopathology. 2001; 34(2): 62-68. 